\title{
Interface stability in stressed solid-phase epitaxial growth
}

\author{
S. Morarka \\ Department of Electrical and Computer Engineering, University of Florida, Gainesville, Florida \\ 32611-6200
}

S. Jin, N. G. Rudawski, ${ }^{\text {a) }}$ and K. S. Jones

Department of Materials Science and Engineering, University of Florida, Gainesville, FL 32611-6400, USA

M. E. Law

Department of Electrical and Computer Engineering, University of Florida, Gainesville, Florida 32611-6200

\section{R. G. Elliman}

Department of Electronic Materials Engineering, Research School of Physical Sciences and Engineering, Australian National University, Canberra, Australian Capital Territory 0200, Australia

(Received 14 January 2011; accepted 17 June 2011; published 11 August 2011)

\begin{abstract}
The role of applied stress on interface stability during $\mathrm{Si}$ solid-phase epitaxial growth was investigated. Transmission electron microscopy observations of growth interface evolution revealed in-plane uniaxial compression (tension) led to interface instability (stability). Additionally, level set simulations revealed that the stress-influenced interface instability was accurately modeled by adjusting the strength of the linear dependence of local interface velocity (rate of change of interface position with respect to time) on local interface curvature proposed in previous work. This behavior is explained in terms of tension in the growth interface controlling interface stability during growth; it is argued that compressive (tensile) stress tends to reduce (enhance) interfacial tension and results in interfacial instability (stability) during growth. () 2011 American Vacuum Society.
\end{abstract}

[DOI: $10.1116 / 1.3610172]$

\section{INTRODUCTION}

The epitaxial amorphous $(\alpha)$ to crystalline phase transformation of $\mathrm{Si}$, known as solid-phase epitaxial growth (SPEG), remains of great technological and fundamental interest. Several decades of study have revealed that many variables influence the kinetics of SPEG including temperature, ${ }^{1,2}$ impurities, ${ }^{3,4}$ and substrate orientation. ${ }^{5}$ There is also interest in studying the role of applied stress on the SPEG process due to the presence of stresses in Si-based device fabrication; ${ }^{6}$ numerous prior studies investigated the influence of different stress states on macroscopic growth kinetics $^{7-14}$ as well as the crystallization of patterned ${ }^{15-19}$ $\alpha$-Si layers. More interestingly, other work ${ }^{11,12,20-22}$ revealed that the stability of the $\alpha /$ crystalline (growth) interface may be altered by the application of macroscopic uniaxial stress applied in the plane of the growth interface, $\sigma_{11}$. Specifically, SPEG with $\sigma_{11}<0$ (compression) was shown to lead to interface instability while $\sigma_{11}>0$ (tension) was shown to produce a stable interface. In principle, for an interface to destabilize (stabilize), the peaks must grow faster (slower) than the troughs, as shown in Fig. 1. However, the underlying mechanism for stress-induced interface instability remains poorly understood. Barvosa-Carter et al. ${ }^{20}$ and Rudawski et $a l .{ }^{11}$ originally attributed stress-influenced stability to differences in the localized stress states at interface peaks $\sigma_{i j}^{p k}$ and troughs $\sigma_{i j}^{t r}$ stemming from a combination of a mismatch in

\footnotetext{
a) Author to whom correspondence should be addressed; electronic mail: ngr@ufl.edu
}

the elastic properties of $\alpha$-Si and crystalline phases ${ }^{24}$ and local interface curvature $\kappa$ variations along a perturbed ${ }^{25}$ interface; the variation between $\sigma_{i j}^{p k}$ and $\sigma_{i j}^{t r}$ leads to variation in the local interface velocity, $v$ (rate of change of interface position with respect to time) at peaks $v_{p k}$ and troughs $v_{t r}$ as per prior studies of macroscopic growth kinetics ${ }^{7-14}$ under applied stress. In prior work, ${ }^{20,26}$ this approach was also used to simulate stressed SPEG evolution, but with somewhat weak experimental confirmation. Interestingly, work by Morarka ${ }^{23}$ determined $\sigma_{i j}^{p k}$ and $\sigma_{i j}^{t r}$ along a wavy interface to be essentially identical to $\sigma_{11}$ (both compressive and tensile) as shown in Figs. 1(b) and 1(c), which does not support the explanation of local stress variations influencing stability. In the absence of applied stress, it is known that a perturbed interface will stabilize, ${ }^{22}$ as shown in Fig. 1(a): specifically, $v$ is retarded (enhanced) where $\kappa<0(\kappa>0)$ at interface peaks (troughs). Therefore, considering the lack of variation in $\sigma_{i j}^{p k}$ and $\sigma_{i j}^{t r}$ compared to $\sigma_{11}$, an apparent synergy between $\sigma_{11}$ and $\kappa$ is controlling interface stability, which contradicts the current understanding ${ }^{11,12,20}$ of stress-influenced interface stability. In this work, stress-induced interface stability is investigated both experimentally and using simulations ultimately providing new insights into interface stability during stressed-SPEG.

\section{EXPERIMENT}

Undoped $50 \mu \mathrm{m}$-thick (001) Si wafers were coated with $\sim 90 \mathrm{~nm}$ of photoresist and patterned using electron beam lithography into $\sim 200$-nm-wide line structures aligned with 


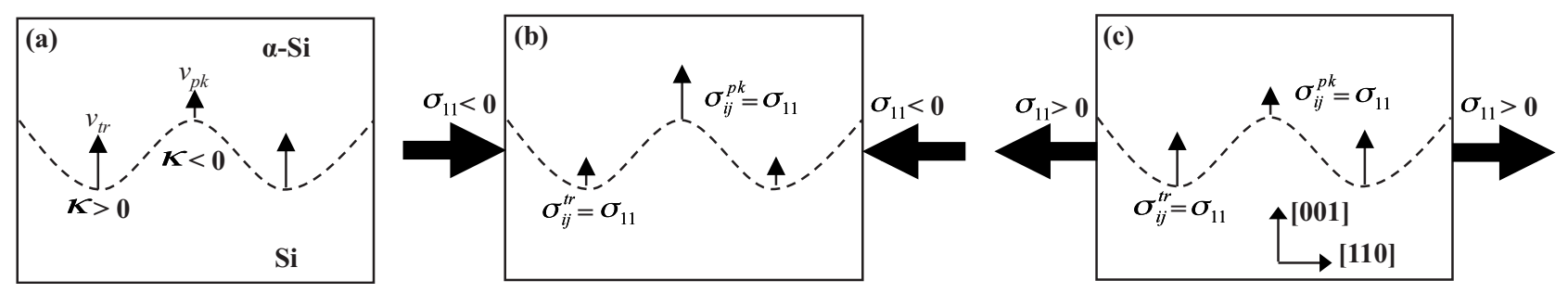

FIG. 1. Influence of macroscopic in-plane uniaxial stress along [110] $\left(\sigma_{11}\right)$ on interface stability during SPEG: (a) with $\sigma_{11}=0$, the local interface velocity at the troughs $\left(v_{t r}\right)$ is faster than at the peaks $\left(v_{p k}\right)$ due to variation in the sign of the local curvature (Ref. 22) $(\kappa)$; (b) with $\sigma_{11}<0$ (compression), the troughs grow slower than the peaks leading to instability; and (c) with $\sigma_{11}>0$ (tension) the troughs grow faster than the peaks stabilizing the interface. Note that in (b) and (c), the local stress states at the troughs, $\sigma_{i j}^{t r}$, and peaks, $\sigma_{i j}^{p k}$, are identical to $\sigma_{11}$ as shown by Morarka (Ref. 23).

the in-plane $[1 \overline{1} 0]$ direction and spaced several hundred $\mathrm{nm}$ apart; this resulted in the line regions being masked by the photoresist. Subsequently, the wafers were reactive ion etched to remove $\sim 100 \mathrm{~nm}$ of exposed Si generating a corrugated wafer surface. The wafers were then $\mathrm{Si}^{-}$-implanted at 20,60 , and $120 \mathrm{keV}$ to doses of $1 \times 10^{15}, 1 \times 10^{15}$, and 3 $\times 10^{15} \mathrm{~cm}^{-2}$ to generate an $\alpha$-Si layer with interface perturbations of similar dimensions to the patterned lines. Uniaxial stress was applied along the in-plane [110] direction up to magnitude of $1.5 \mathrm{GPa}$ using a method described elsewhere. ${ }^{10,12}$ The samples were then annealed at temperature $T=525{ }^{\circ} \mathrm{C}$ for up to $4.0 \mathrm{~h}$ (individual stress-free, compressive, and tensile specimens were annealed simultaneously). Cross-sectional transmission electron microscopy (XTEM) was used to image the SPEG evolution with samples prepared using site-specific focused ion beam (FIB) milling. As in prior work, the SPEG process was modeled using level set techniques ${ }^{27-29}$ and implemented in the Florida Object-Oriented Process Simulator as described elsewhere. ${ }^{30}$

\section{RESULTS AND DISCUSSION}

In prior work, Morarka et $a .^{28}$ were able to accurately simulate the stress-free two-dimensional SPEG process using level set methods ${ }^{30}$ by modeling the local interface velocity as a function of both local curvature and the angle of deviation of the local interface normal away from the [001] direction toward [110], $\theta$, via

$$
v(\kappa, \theta)=\left(1+A_{\kappa} \kappa\right) f(\theta) v_{[001]},
$$

where $A_{\kappa}$ is the curvature parameter, $f(\theta)$ is the orientationdependent growth velocity ${ }^{5}$ normalized to (001)-oriented growth, and $v_{[001]}$ is the (001)-oriented growth velocity. Note that $f(\theta)$ is $T$ independent ${ }^{5}$ and the $T$ dependence ${ }^{1,2}$ of $v_{[001]}$ is accounted for in all presented simulations. A value of $A_{\kappa}$ $=2.0 \times 10^{-7} \mathrm{~cm}$ accurately modeled the stress-free twodimensional SPEG process. However, it is instructive to observe the effect of varying $A_{\kappa}$ on the simulated SPEG evolution. Figure 2 presents the simulated SPEG evolution of a structure with an initially wavy growth interface after annealing at $T=525{ }^{\circ} \mathrm{C}$ for $2.0 \mathrm{~h}$ with different values of $A_{\kappa}$. For the case of $A_{\kappa}=0$, the resulting growth interface is rougher and more perturbed than the initial interface. With the curvature parameter increased to $A_{\kappa}=1.5 \times 10^{-7} \mathrm{~cm}$, the interface remains rough and perturbed after growth, but less so than the case of $A_{\kappa}=0$. Finally, with $A_{\kappa}=4.0 \times 10^{-7} \mathrm{~cm}$, the perturbations still remain but have decreased greatly in magnitude compared to the cases of $A_{\kappa}=0$ or $A_{\kappa}=1.5 \times 10^{-7} \mathrm{~cm}$. It is very interesting that simulated SPEG with lower values of $A_{\kappa}$ are very similar to observations of interface instability with $\sigma_{11}<0$ while simulations with larger values of $A_{\kappa}$ are similar to observations of stable interfaces with $\sigma_{11} \geq 0$. Thus, the result that changing $A_{\kappa}$ can apparently control simulated interface instability suggests that the role of applied $\sigma_{11}$ on SPEG evolution may be accounted for simply by changing the curvature parameter.

Figure 3 shows a sequence of XTEM micrographs of the SPEG evolution in the previously described test structure under different applied $\sigma_{11}$. The as-implanted structure, shown in Fig. 3(a), exhibits a corrugated surface with an initial growth interface having perturbations $\sim 100 \mathrm{~nm}$ in amplitude and $\sim 200 \mathrm{~nm}$ in width with the corresponding simulated as-implanted structure shown in Fig. 3(b). After annealing at $T=525^{\circ} \mathrm{C}$ for $4.0 \mathrm{~h}$ with $\sigma_{11}=-0.5 \mathrm{GPa}$, the initial perturbation has increased in amplitude and the initially flat portions of the growth interface have roughened greatly. In contrast, after annealing at $T=525{ }^{\circ} \mathrm{C}$ for $2.0 \mathrm{~h}$ with $\sigma_{11}=0.5 \mathrm{GPa}$, the initial perturbation has greatly decreased in amplitude and the initially flat portions of the growth interface have remained stable. For specimens annealed with $\sigma_{11}>0$, the evolution was essentially indistinguishable from the stress-free case, so only one case of $\sigma_{11}$ $>0$ is provided for clarity. Note that the $\sigma_{11}<0$ sample was annealed for twice as long as the $\sigma_{11}<0$ case; this was done

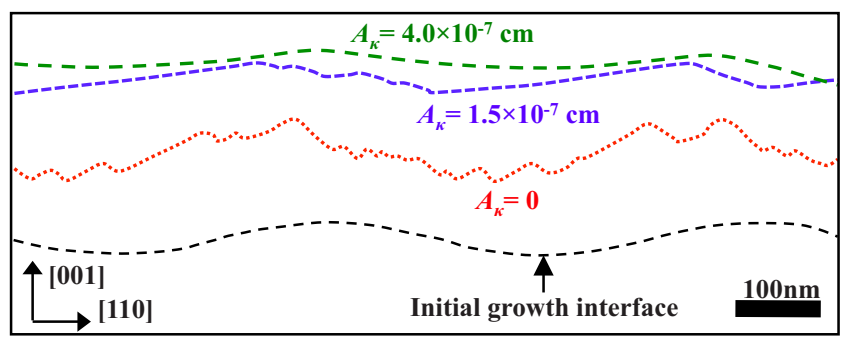

FIG. 2. (Color online) Influence of varying the curvature parameter, $A_{\kappa}$, on the SPEG interface evolution of a structure with a wavy initial growth interface at $T=525^{\circ} \mathrm{C}$ after $2.0 \mathrm{~h}$ of annealing as simulated using Eq. (1) with $A_{\kappa}=0(-), 1.5 \times 10^{-7}(-)$, and $4.0 \times 10^{-7} \mathrm{~cm}(-)$. Note that interface stability increases with increasing $A_{\kappa}$. 


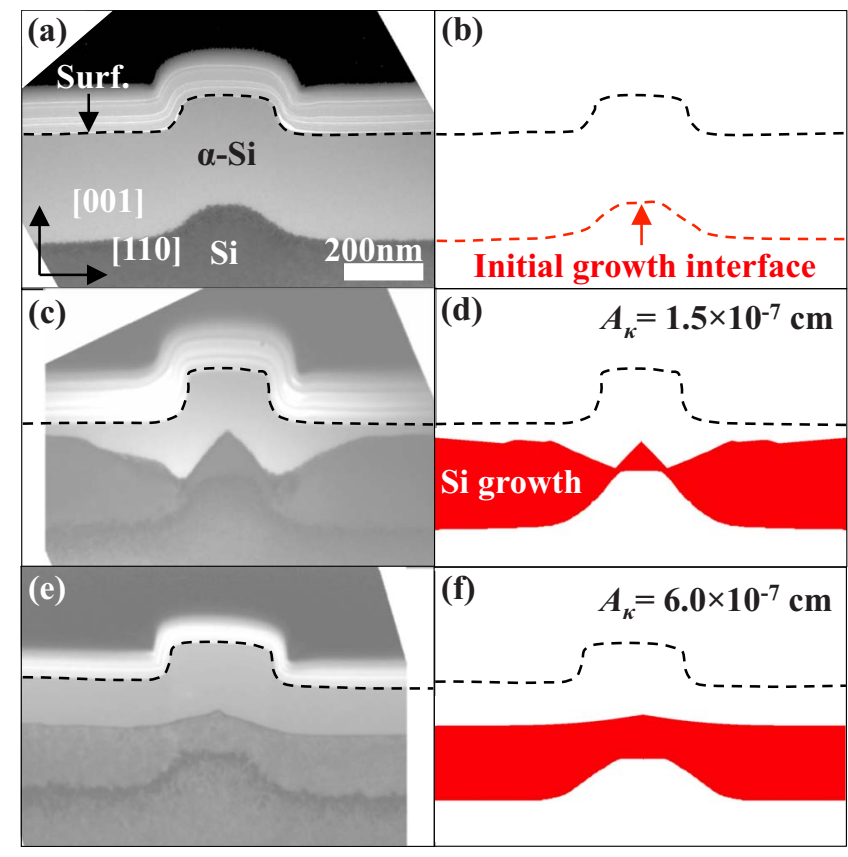

FIG. 3. (Color online) Observed and simulated [using Eq. (1)] stressedSPEG evolution with applied in-plane uniaxial stress $\left(\sigma_{11}\right)$ at $T=525{ }^{\circ} \mathrm{C}$ : (a) XTEM image of the as-implanted structure and (b) the simulated asimplanted structure. (c) XTEM image of the structure after $4.0 \mathrm{~h}$ of annealing with $\sigma_{11}=-0.5 \mathrm{GPa}$ (compression) and (d) the corresponding simulated SPEG evolution with a curvature parameter $\left(A_{\kappa}\right)$ of $1.5 \times 10^{-7} \mathrm{~cm}$. (e) XTEM image of the structure after $2.0 \mathrm{~h}$ of annealing with $\sigma_{11}=0.5 \mathrm{GPa}$ (tension) and (f) the corresponding simulated SPEG evolution with $A_{\kappa}$ $=6.0 \times 10^{-7} \mathrm{~cm}$. Note that stress-free SPEG evolution was essentially identical to SPEG evolution with $\sigma_{11}>0$.

to account for reports ${ }^{11,12}$ that the macroscopic growth kinetics with $\sigma_{11}<0$ are slower than the kinetics with $\sigma_{11} \geq 0$ by a factor of $\sim 2$. This allowed the SPEG evolution in samples with $\sigma_{11}<0$ and $\sigma_{11} \geq 0$ to be compared after approximately the same level of completion.

Figure 3(d) shows the SPEG evolution at $T=525{ }^{\circ} \mathrm{C}$ for $4.0 \mathrm{~h}$ simulated using Eq. (1) with $A_{\kappa}=1.5 \times 10^{-7} \mathrm{~cm}$. The simulation accurately accounts for the general evolution of the growth interface, although the magnitude of the center perturbation is smaller than experimentally observed. In contrast, Fig. 3(f) shows the SPEG evolution at $T=525{ }^{\circ} \mathrm{C}$ for $2.0 \mathrm{~h}$ simulated using Eq. (1) with $A_{\kappa}=6.0 \times 10^{-7} \mathrm{~cm}$. Similarly, the simulation accurately replicates the experimentally observed evolution where the magnitude of the initial perturbation has decreased and the initially flat portions of the interface remain stable. In both cases of Figs. 3(d) and 3(f), the values of $A_{\kappa}$ were chosen to provide the best visual fit to the observed XTEM images.

As predicted by the simulations in Fig. 2 and confirmed experimentally in Fig. 3, the evolution of a growth interface during SPEG was effectively modeled using Eq. (1) and varying the magnitude of $A_{\kappa}$. However, it is not well understood why SPEG evolution without applied stress can be modeled effectively using the linear dependence of the local interface velocity with local curvature presented Eq. (1). One possibility that was discussed in prior work ${ }^{28}$ studying stressfree SPEG was that the linear dependence of $v$ with $\kappa$ is related to the presence of interfacial tension, $\gamma$, (or, interchangeably, energy) between $\alpha$ and crystalline phases which arises due to bond distortions ${ }^{31,32}$ across the interface. If it is assumed that the presence of $\gamma$ provides an inherent driving force for interface stabilization, then applying external $\sigma_{11}$ $>0$ simply increases this driving force. However, in the case of $\sigma_{11}<0, \gamma$ is effectively counteracted and the driving force for interface stabilization is reduced or removed, effectively reducing growth to being controlled mostly by substrate orientation dependence. ${ }^{5}$ Of course, this does not provide any explanation for why the presence of $\gamma$ stabilizes the interface. Phan et al. ${ }^{26}$ originally advanced $\gamma$ dependence of the local interface velocity of the form

$$
v(\kappa) \propto 1-\exp \left(\frac{\Delta G_{\alpha-c}-\gamma \kappa \Omega}{k T}\right),
$$

where $\Delta G_{a-c}$ is the free energy difference between amorphous and crystalline phases, $\Omega$ is the atomic volume of $\mathrm{Si}$, and $k T$ has the usual meaning. This approach was based on minimization of excess interfacial free energy to produce the greatest free energy driving force for crystallization. However, extensive work by Morarka et al. ${ }^{27,28}$ determined that this approach was wholly incapable of accounting for stressfree interface evolution with calculations also showing the proportionality in Eq. (2) to be exceedingly close to unity for a wide range of $\kappa$. This suggests that if $\gamma$ is controlling instability, it is due to kinetic, rather than thermodynamic, considerations, though the exact nature of these kinetic considerations is still not understood. As per prior atomistic models of growth, this may be related to in-plane migration of island ledges being influenced by $\gamma$. In prior work, Rudawski et al. ${ }^{11,12,33}$ suggested that migration was enhanced (retarded) by $\sigma_{11}>0\left(\sigma_{11}<0\right)$; therefore, it may be the case that applying $\sigma_{11}$ simply increases or counteracts the contribution of $\gamma$ toward migration enhancement.

It is also intriguing to note that the simulated extent of crystallization with $A_{\kappa}=0$ is approximately half that simulated with $A_{\kappa}=4.0 \times 10^{-7} \mathrm{~cm}$, as shown in Fig. 2; this is strikingly similar to observations from Rudawski et al. ${ }^{11,12,33}$ revealing that macroscopic growth kinetics with $\sigma_{11} \geq 0$ were approximately twice as fast as compared to $\sigma_{11}<0$. This behavior was attributed to stress-altered ledge migration, but it may be the case that this occurs as a result of the effective alteration of $\gamma$ with applied stress (at least for the very specific case of in-plane uniaxial stress); reducing $A_{\kappa}$ sufficiently effectively forces SPEG evolution to be controlled entirely by orientation dependence and the resulting anisotropy ${ }^{5}$ in $v$ leads to an overall reduction in growth kinetics.

\section{SUMMARY AND CONCLUSIONS}

In conclusion, the role of applied in-plane uniaxial stress on interface instability during solid-phase epitaxial growth of $\mathrm{Si}$ was investigated. It was revealed that compression (tension) led to interface instability (stability). This behavior was accurately modeled by adjusting the strength of the linear dependence of the local interface velocity on local interface 
curvature. Physically, it was argued that interfacial tension between the amorphous and crystalline interface tends to stabilize the interface during growth. Therefore, it was advanced that applied external in-plane uniaxial compressive (tensile) stress may reduce (enhanced) this interfacial tension, leading to instability (stability).

\section{ACKNOWLEDGMENTS}

The authors acknowledge the Semiconductor Research Corporation for funding this work. The Major Analytical Instrumentation Center at the University of Florida is acknowledged for use of the FIB and TEM facilities.

${ }^{1}$ J. M. Poate et al., Nucl. Instrum. Methods Phys. Res. B 55, 533 (1991).

${ }^{2}$ J. A. Roth, G. L. Olson, D. C. Jacobson, and J. M. Poate, Appl. Phys. Lett. 57, 1340 (1990).

${ }^{3}$ E. F. Kennedy, L. Csepregi, J. W. Mayer, and T. W. Sigmon, J. Appl. Phys. 48, 4241 (1977).

${ }^{4}$ B. C. Johnson and J. C. McCallum, Phys. Rev. B 76, 045216 (2007).

${ }^{5}$ L. Csepregi, E. F. Kennedy, J. W. Mayer, and T. W. Sigmon, J. Appl. Phys. 49, 3906 (1978).

${ }^{6}$ S. M. Hu, J. Appl. Phys. 70, R53 (1991).

${ }^{7}$ M. J. Aziz, P. C. Sabin, and G. Q. Lu, Phys. Rev. B 44, 9812 (1991).

${ }^{8}$ G. Q. Lu, E. Nygren, and M. J. Aziz, J. Appl. Phys. 70, 5323 (1991).

${ }^{9}$ E. Nygren, M. J. Aziz, D. Turnbull, J. M. Poate, D. C. Jacobson, and R. Hull, Appl. Phys. Lett. 47, 232 (1985).

${ }^{10}$ N. G. Rudawski, K. S. Jones, and R. Gwilliam, Appl. Phys. Lett. 91, 172103 (2007).

${ }^{11}$ N. G. Rudawski, K. S. Jones, and R. Gwilliam, Phys. Rev. Lett. 100, 165501 (2008).

${ }^{12}$ N. G. Rudawski, K. S. Jones, and R. Gwilliam, Mater. Sci. Eng., R. 61, 40 (2008).
${ }^{13}$ N. G. Rudawski, K. S. Jones, and R. Gwilliam, Appl. Phys. Lett. 92, 232110 (2008).

${ }^{14}$ N. G. Rudawski, K. S. Jones, and R. Gwilliam, J. Mater. Res. 24, 305 (2009).

${ }^{15}$ C. R. Olson, E. Kuryliw, B. E. Jones, and K. S. Jones, J. Vac. Sci. Technol. B 24, 446 (2006)

${ }^{16}$ N. G. Rudawski, K. S. Jones, and R. G. Elliman, J. Vac. Sci. Technol. B 26, 435 (2008).

${ }^{17}$ N. G. Rudawski, K. S. Jones, S. Morarka, M. E. Law, and R. G. Elliman, J. Appl. Phys. 105, 081101 (2009).

${ }^{18}$ N. G. Rudawski, K. N. Siebein, and K. S. Jones, Appl. Phys. Lett. 89, 082107 (2006).

${ }^{19}$ Y. G. Shin, J. Y. Lee, M. H. Park, and H. K. Kang, Jpn. J. Appl. Phys., Part 1 40, 6192 (2001).

${ }^{20}$ W. Barvosa-Carter, M. J. Aziz, L. J. Gray, and T. Kaplan, Phys. Rev. Lett. 81, 1445 (1998).

${ }^{21}$ W. Barvosa-Carter, M. J. Aziz, A. V. Phan, T. Kaplan, and L. J. Gray, J. Appl. Phys. 96, 5462 (2004).

${ }^{22}$ J. F. Sage, W. Barvosa-Carter, and M. J. Aziz, Appl. Phys. Lett. 77, 516 (2000).

${ }^{23}$ S. Morarka, Ph.D. thesis, University of Florida, 2010.

${ }^{24}$ A. Witvrouw and F. Spaepen, J. Appl. Phys. 74, 7154 (1993).

${ }^{25}$ R. J. Asaro and W. A. Tiller, Metall. Trans. 3, 1789 (1972).

${ }^{26}$ A. V. Phan, T. Kaplan, L. J. Gray, D. Adalsteinsson, J. A. Sethian, W. Barvosa-Carter, and M. J. Aziz, Model. Simul. Mater. Sci. Eng. 9, 309 (2001).

${ }^{27}$ S. Morarka, N. G. Rudawski, and M. E. Law, J. Vac. Sci. Technol. B 26, 357 (2008).

${ }^{28}$ S. Morarka, N. G. Rudawski, M. E. Law, K. S. Jones, and R. G. Elliman, J. Appl. Phys. 105, 053701 (2009).

${ }^{29}$ S. Morarka, N. G. Rudawski, M. E. Law, K. S. Jones, and R. G. Elliman, J. Vac. Sci. Technol. B 28, C1F1 (2010).

${ }^{30}$ M. E. Law and S. M. Cea, Comput. Mater. Sci. 12, 289 (1998).

${ }^{31}$ N. Bernstein, M. J. Aziz, and E. Kaxiras, Phys. Rev. B 58, 4579 (1998).

${ }^{32}$ F. Spaepen, Acta Metall. 26, 1167 (1978).

${ }^{33}$ N. G. Rudawski and K. S. Jones, Scr. Mater. 61, 327 (2009). 